\title{
Alexa Mohl \\ Im Osten Nichts Neues? \\ Der Leninismus in Bahros Konzept der Kulturrevolution
}

Elemente leninistischen Denkens sind in Rudolf Bahros, ,Kritik des real existierenden Sozialismus" leicht auszumachen: Von beschränkten Möglichkeiten der unmittelbaren Produzenten, systemkritisches Bewußtsein auszubilden und von daraus folgenden führenden Aufgaben eines neuen Bundes der Kommunisten für eine kulturrevolutionäre Umwälzung ist die Rede. In seinem ,Sozialismus in keinem Land ${ }^{66}$ überschriebenen Diskussionsbeitrag zur ,Alternative" kennzeichnet Detlev Claussen Bahros Vorstellungen zur Organisation der Kulturrevolution, die sich an der Parteitheorie Lenins orientiert, als Denksperre, die Bahro jedoch nicht persönlich anzulasten sei, zumal diese Vorstellung mit seinen theoretischen Ansätzen in Widerspruch geraten müsse (1). Zudem sei diese Parteikonzeption das einzige traditionelle Moment in Bahros Argumentation (2).

Es fehlt aber nicht an Diskussionsbeiträgen zu Bahros ,Alternative" Verhaftetsein in traditionellen Denkmustern anders bewerten: Unter dem TiteI „Auflösung und Wiederkehr des Leninismus" behandelt Günter Maschke die neue marxistische Opposition in den sozialistischen Ländern des Ostblocks (3). Rudolf Bahro wird in diesem Artikel ausschließlich als Leninist wahrgenommen, ais einer derjenigen oppositionellen Köpfe, die vorgeben, emanzipatorischen Bedürfnissen der Massen zum gesellschaftlichen Durchbruch verhelfen zu wollen, in Wirklichkeit aber den ,Herrschaftsanspruch" ${ }^{6}$ einer neuen, intellektuellen Avantgarde ${ }^{65}$ formulierten. Diktiert vom Haß auf den alten Parteiapparat, der jedoch nicht aus emanzipatorischen Interessen fließe, sondem in der Tatsache begründet liege, daß der ,bestehende Apparat" auf diese Intellektuellen, „verzichten " könne, würden die Vorschläge Bahros zur Organisation und Strategie der Kulturrevolution nur auf eine neue Diktatur hinauslaufen - auf eine Erziehungsdiktatur, die Maschke für die ,beliebteste Diktatur wohlmeinend-ohnmächtiger Revolutionäre" hält. Sein Fazic: „Die von Bahro attackierte Inquisition wäre sofort wieder da." (4)

Im Osten wirklich nichts Neues? - Ich möchte in diesem Beitrag der Frage nachgehen, welche Bedeutung den leninistischen Denkstrukturen Bahros für sein Konzept der Kulturrevolution zukommt.

\footnotetext{
1 Detlev Claussen: Sozialismus in keinem Land, in: links, sozialistische Zeitung Nr. 95, Januar 1978, S. 16

2 ebenda

3 Günter Maschke: Auflösung und Wiederkehr des Leninismus, in: FAZ vom 21. 101978

4 ebenda
} 


\section{Die Analyse des real existierenden Sozialismus und das strategische Konzept der Kulturrevolution}

„Die russische sozialistische Bewegung des Jahrhundertanfangs (fand) objektiv eine andere Aufgabe zu erfüllen vor ..., als die, zu der sie sich berufen glaubte." (5) Dies ist die Hauptthese von Bahros Analyse des real existierenden Sozialismus. Die Aufgabe der Oktoberrevolution, war noch nicht der Sozialismus, so aufrichtig die Bolschewiki daran glaubten, sondern die schnelle industrielle Entwicklung Rußlands auf einem nichtkapitalistischen Weg“ (58). Da aber ein vorkapitalistisches Land mit halbfeudalen, halbasiatischen Strukturen und Produktionsweisen sich ohne das Verhältnis von Lohnarbeit und Kapital nicht industrialisieren könne, bedurfte es einer anderen Instanz, um die fehlenden ökonomischen Grundlagen für den Sozialismus zu schaffen. An die Stelle des Kapitals trat der ,Staat als Zuchtmeister der Gesellschaft für ihre technische und soziale Modernisierung “6 (150).

Angesichts der vorrangigen Aufgabe, die Produktivkräfte zu entwickeln und der strukturellen Notwendigkeit, den Staatsapparat als Motor und Dirigenten dieses Entwicklungsprozesses einzusetzen, gerieten die ursprünglichen Ziele der intendierten sozialistischen Revolution aus dem Blickfeld. Standen sie doch teilweise in direktem Gegensatz zur Industrialisierung. Die Arbeitsteilung konnte nicht nur nicht aufgehoben werden, sie mußte erst einmal entwickelt werden. Damit erwiesen sich alle Marxschen Antizipationen des Reichs der Freiheit, die Selbstbetätigung und Selbstverwirklichung des Menschen, die Aneignung aller seiner Wesenskräfte als zunächst illusorisch. Auch die Entfremdung der Arbeit habe deshalb nicht aufgehoben werden können. lst sie doch Bahro zufolge nicht nur auf den Warenfetischismus zurückzuführen, sondern in der Arbeitsteilung schlechthin verwurzelt (vgl. 167). Und die Arbeitsteilung sei auch das ,Fundament der Klassengesellschaft" . ,Gerade die russische Revolution ist geeignet", so Bahro, ,uns nachdrücklich zu belehren, daß die Zeit der Arbeitsteilung auch die Zeit der Herrschaft des Menschen über den Menschen ist." (168).

Die Vorbedingungen für die allgemein menschliche Emanzipation sind aber Bahro zufolge jetzt gegeben. Der technisch-ökonomische Fortschritt in den Ländern des real existierenden Sozialismus habe ein Niveau erreicht, das den Kampf um den Sozialismus auf die Tagesordnung setze (vg1. 58). Und nicht nur die objektiven Bedingungen seiner Möglichkeit seien voll ausgebildet. Der Sozialismus sei auch objektiv notwendig geworden: „Die Ablösung der politbürokratischen Diktatur ist sozialökonomisch überfällig geworden" (386). Die im Rahmen der bestehenden Produktionsweise ,unlösbaren Widersprüche des materiellen Lebens und insbesondere der technischen Basis" verlangten immer drängender nach einer radikalen ökonomischen Alternative (vgl. 426). Auch die subjektiven Bedingungen seien gegeben. Die Entwicklung der Produktivkräfte habe ein ,überschüssiges Bewußtsein " herausgebildet (vgl. 304). Dieser ,menschliche Qualifikationsüberschuß ${ }^{66}$ (206), der entstanden sei, weil die Technologie gebildete Massen erfordere, diese Qualifikationen aber in der Arbeit nicht voll absorbiert werden oder im Konsum kompensiert wer-

5 Rudolf Bahro: Die Alternative. Zur Kritik des real existierenden Sozialismus. Köln, Frankfurt am Main 1977, S. 23. Im Folgenden belege ich Zitate und Anlehnungen aus Bahros Schrift im Text. Die Ziffern in den Klammern geben, wenn ihnen nicht eine Fufsnote auf der gleichen Seite entspricht, die Seitenzahlen in Bahros „Die Alternative" an. 
den könnten, setze sich in emanzipatorische Interessen um, die sich gegen die Bevormundung der Gesellschaft durch den Staat, gegen das Privileg der Meinungsbildung und Entscheidung über die allgemeinen Angelegenheiten, die bürokratische Form der Verfügung über die Produktivkräfte und über den ganzen sozialen Lebensprozeß richten (vgl. $371 \mathrm{f}$.). Bahro sieht in diesem Antagonismus einen „Fortschritt der Weltgeschichte" weil darin zum Ausdruck komme, „daß das Ringen in den industriell entwickelten Ländern bereits in der Sphäre der eigentlichen menschlichen Wesenskräfte ausgetragen wird, nicht mehr hauptsächlich um Leib- und Magen-, Kleidungs- und Wohnungsfragen" gehe (376).

Diesen emanzipatorischen Interessen ständen aber die verselbständigten Interessen des Apparats auf Erhaltung des politischen status quo entgegen. Die Entwicklung in den Ländern des real existierenden Sozialismus habe dahin geführt, daß der politische Überbau selbst sich ,,in Teufelskreis der alten Arbeitsteilung festgefahren hat" (179). Aufgrund seiner monolithischen Konstruktion und mechanistischen Arbeitsweise sei das ganze System der politischen Institutionen unfähig zu aktiver Selbstveränderung (vgl. 427). Als eine vom Volk, von den Massen total isolierte Maschine könne sie auch nicht mehr integral funktionieren: ,... sie muß der Gesellschaft von außen und oben einen Konsensus aufzwingen, der dann nichts als der alte besondere Staats- und Kirchengeist ist, der sich im Zustand der Macht und Gnade halten möchte" (425). Bahro spricht sogar von einem neurotischen Funktionieren des Parteiapparats im real existierenden Sozialismus, da dieser nicht mehr garantiere, daß sich „die Gesellschaft institutionell regelmäßig und rechtzeitig an die Veränderungen ihrer selbst und ihrer Umwelt anpaßt" (291).

Deshalb sei eine neue, eine andere kommunistische Partei notwendig, die Bahro zur besseren Unterscheidung von den herrschenden kommunistischen Parteien „Bund der Kommunisten“ nennt (vgl. 409). Diese neuzuformierende politische Macht werde aus Individuen bestehen, die aufgrund ihres emanzipatorischen Bewußtseins, ihrer durch Ausbildung hervorgebrachten und durch ihre Position im Gesamtarbeitsprozeß geschulten intellektuellen synthetischen Fähigkeiten in eine oppositionelle Position gegen die Bürokratie geraten seien und sich entschieden gegen die Fortsetzung der alten gesellschaftlichen Arbeitsteilung, die Subalternität weiter Kreise der unmittelbaren Produzenten und gegen die Gemeinschaftslosigkeit des alltäglichen gesellschaftlichen Lebens wendeten. Diese unzufriedenen Ideologen mit angestauten Kräften für gesellschaftliches Engagement, die sich im status quo nicht entfalten können, werden sich nach Bahro um die Idee der allgemeinen Emanzipation als ,,kollektive Intellek tuelle" (430) im Bund der Kommunisten vereinigen. Den Begriff des kollektiven Intellektuellen hält Bahro für ,die Quintessenz aller Ideen über die politische Führungsfunktion und innere Verfassung der Kommunistischen Partei, wie sie von Marx und Engels über den jungen Lenin, über Rosa Luxemburg und Antonio Gramsci bis zu den heutigen marxistischen Denkansätzen herausgearbeitet wurden“"(431). Der Bund der Kommunisten soll einen subjektiven Resonanzboden für eine wirklich kommunistische Alternative darstellen. Er soll die Gegenkräfte formieren und in Bewegung setzen, um gesell- 
schaftliche Verhältnisse zu schaffen, in denen die Subaltemität, d. h. die Entfremdung der Menschen vom Gemeinwesen verschwindet.

\section{Die These vom reduzierten Bewußtsein der unmittelbaren Produzenten}

Warum eine neue kommunistische Partei? Hat der Kritiker mit seiner These von der „Neuauflage des Leninismus" (6) doch recht? Kehrt der ,klassische Leninismus, (nachdem er) sich transformierte in die Herrschaft des Apparats ..., verändert wieder im Plan, aufgeklärter Subjekte, die die anderen mit sich reißen ...?"(7)

Hinter Bahros Konzept der Kulturrevolution steht die Auffassung, daß der „Mechanismus des Fortschritts" von einer Gesellschaftsformation zur anderen nicht von einem Pol der alten Gesellschaft, den unterdrückten Klassen, erzeugt wird. Es sei, das habe die Psychologie seit Freud gezeigt, unmöglich, „daß eine unterdrückte, der Arbeitsteilung unterworfene, entfremdete Klasse von unmittelbaren Produzenten ,selbst ${ }^{6}$ herrschende Klasse werden und in dieser Rolle die Hegemonie über den ganzen Kulturprozeß ihrer Gesellschaft ausüben könnte" (235). Die Momente des Fortschritts gingen vielmehr aus der Ganzheit des widerspruchsvollen sozialen Sy. stems hervor. Nicht die Zuspitzung der abstrakten Widersprüche und die Empörung der Unterdrückten eröffneten die neue Perspektive, sondern die „Formierung eines strukturierten Organismus, in dem sich mehrere Elemente der alten Gesellschaft zu einer neuen Qualität kombinieren" (175). Nur ein Bündnis solcher Individuen, die bereits in der alten Gesellschaftsordnung eine besondere intellektuelle Qualifikation für synthetische Arbeit erworben haben und diese im gesellschaftlichen Produktions- und Lebensprozeß anzuwenden in der Lage waren (vgl. $171 \mathrm{f}$.), die damit die Voraussetzung zur Antizipation und Organisation einer neuen sozialen Synthesis mitbringen, könnte die Geschichte vorwärtstreiben.

Diese Voraussetzungen seien aber bei den bisher unterdriickten und in ihrem realen Lebensprozeß von der gesellschaftlichen Synthesis der alten Ordnung ausgeschlossenen Klassen nicht gegeben. Es sei eine allgemeine historische Erfahrung, "daß die werktätigen Massen ... vorwiegend die quantitative Akkumulation leisten ${ }^{66}$ (175). In keinem bekannten historischen Falle sei der erste schöpferische Impuls für eine neue Kultur von ihnen ausgegangen (vgl. ebenda). ,Die unmittelbaren Bedürfnisse der subalternen Schichten und Klassen sind immer konservativ, antizipieren in Wirklichkeit nie positiv eine neue Lebensform" (174). Dies gilt nach Bahro auch für die unmittelbaren Produzenten in den Ländern des real existierenden Sozialismus: „Die bisherige klassengesellschaftliche Arbeitsteilung läuft eben darauf hinaus, daß die überwältigende Mehrheit der Individuen entscheidend durch die früh vorprogrammierte Unterordnung unter eine bestimmte hauptberufliche Tätigkeit geprägt und beschränkt wird, so daß sie dann höchstens passiv an anderen Sphären partizipieren kann" (207). Die Subaltemität, und das heißt bei Bahro nichts anderes als gerade das Ausgeschlossensein vom Gemeinwesen, der gesellschaftlichen Synthesis,

\footnotetext{
$6 \quad$ Maschke, a.a.O.

7 ebenda
} 
werde nicht nur in der Arbeit produziert (vgl. $208 \mathrm{f}$.). Schon die frühkindliche Sozialisation stehe unter dem Diktat der herrschenden Arbeitsteilung und ihrer geplanten Reproduktion, fördere also nicht die Anlagen und Talente, wie sie natürlicherweise vorhanden seien, sondem nur in dem Maße und der Intensität, wie der gesellschaftliche Produktionsprozeß sie erfordere (vgl. 209). Dieser Prozeß der "Unterentwicklung der Lernmotivation schon bei Kindern der benachteiligten Schichten " (210) werde im Ausbildungssystem fortgesetzt. Unvermeidliche Reaktion darauf, daß die Gesellschaft die Entfaltung, Entwicklung und Betätigung zahlloser Menschen frühzeitig beschränkt und blockiert, sei die Ausbildung von kompensatorischen Interessen, die mit Ersatzbefriedigungen abgespeist würden (vgl. 322).

Die hier versammelten „Stellen“ aus Bahros Argurnentation belegen m. E. in der Tat nachdrücklich, daß der Autor die leninistische These vom ,induzierten Klassenbewußtsein" vertritt. Bahro geht sogar so weit, die Marxsche Theorie in diesem Punkt mit dem Leninismus zu ídentifizieren. Seinem Verständnis nach ,kündigt sich bereits in dem (in den Marxschen Frühschriften formulierten) Verhältnis von ,Philosophie ' und ,Proletariat ‘... eine Gesellschaft an, die - in der Sprache der Feuerbachthesen - mit Notwendigkeit dahin kommt, sich, in zwei Teile zu sondern, von denen der eine über die Geselischaft erhaben ist". Denn die ,Philosophie " das können nur die "Philosophen " mit ihrem intellektuell-erzieherischen und administrativen Anhang sein!" (230).

Marx und Engels aber teilten die Vorstellung, die unmittelbaren Bedürfnisse der subalternen Schichten und Klassen seien immer konservativ, die Massen antizipierten nie positiv eine qualitativ neue Lebensform (vgl. 174 f.) durchaus nicht, schon gar nicht in der Verallgemeinerung, mit der Bahro sie vorträgt (175). Marx und Engels setzten ihre revolutionären Hoffnungen auf die Arbeiterklasse, weil in deren Kämpfen ein gesellschaftlich emanzipatorisches Handeln sichtbar wurde, welches nicht eine bessere Position innerhalb der bürgerlichen Gesellschaftsordnung anstrebte, sondern diese qualitativ zu überwinden trachtete. An einem 1844 an Feuerbach gerichteten Brief läßt sich dieses Vertrauen von Marx in die intellektuellen und moralischen Fähigkeiten für emanzipative Praxis bei Arbeitern und ihr lebhaftes Interesse an qualitativer gesellschaftlicher Veränderung ahlesen. Dort schildert Marx seine Erfahrungen aus Versammlungen französischer Arbeiter: „Sie müßten einer der Versammlungen der französischen ouvriers beigewohnt haben, um an die jungfräuliche Frische, an den Adel, der unter diesen abgearbeiteten Menschen hervorbricht, glauben zu können. Der englische Proletarier macht auch Riesenfortschritte, aber es fehlt ihm der Kulturcharakter der Franzosen. Ich darf aber nicht vergessen, die theoretischen Verdienste der deutschen Handwerker in der Schweiz, London und Paris hervorzuheben. Nur ist der deutsche Handwerker noch zu viel Handwerker. Jedenfalls aber bereitet die Geschichte unter diesen ,Barbaren" das praktische Element zur Emanzipation des Menschen vor" (8). Auch in der „Heiligen Familie“" finden sich Belege dafür, daß Marx und Engels den Arbeitern die Fähigkeit, neue Lebensformen zu antizipieren, durchaus nicht absprachen. Das Gegenteil ist richtig,

8 Marx an Ludwig Feuerbach vom 11. August 1844, MEW 27, S. 426 
wie folgendes Urteil zeigt: „Man muß das Studium, die Wißbegierde, die sittliche Energie, den rastlosen Entwicklungstrieb der französischen und englischen Ouvriers kennengeleint haben, um sich von dem menschlichen Adel dieser Bewegung eine Vorstellung machen zu können. ${ }^{\text {" }}$ (9) Und: ,... die englischen und französischen Arbeiter (haben) Assoziationen gebildet, in welchen nicht nur ihre unmittelbaren Bedürfnisse als Arbeiter, sondern ihre Bedürfnisse als Menschen den Gegenstand ihrer wechselseitigen Belehrung bilden, worin sie überdem ein sehr gründliches und umfassendes Bewußtsein über die ,ungeheure' und ,unermeßbare' Kraft äußern, welche aus ihrem Zusammenwirken entsteht. " (10)

Marx und Engels vertrauten also auf eine praktische gesellschaftskritische Eigeninitiative der Arbeiterklasse. Das zeigt auch eine „Stelle" aus dem „Kommunistischen Manifest", wo den utopischen Sozialisten angekreidet wird, sie erblickten ,auf der Seite des Proletariats keine geschichtliche Selbsttätigkeit, keine ihm eigentümliche politische Bewegung" (11). Marx geht davon aus, daß die Welt längst den Traum von der Sache besitzt, von der die kritische Philosophie ihr nur das gesellschaftlich adäquate Bewußtsein vermitteln muß, indem sie ihre eigenen Aktionen ihr erklärt (12). Die sozialistischen Theoretiker hatten es mit den beginnenden proletarischen Kämpfen nicht mehr nötig, die Wissenschaft, d.h. die Bedingungen der gesellschaftlichen Emanzipation, in ihren Köpfen zu suchen. Sie brauchten sich nur noch Rechenschaft abzulegen von dem, was sich vor ihren Augen abspielte und sich zum Organ desselben zu machen (13). Sie konnten nun im Gegensatz zu den utopischen Sozialisten ihre „Wissenschaft auf die Erkenntnis der vom Volk selbst gemachten sozialen Bewegung beschränken" (14).

Daß Marx und Engels die Bedingungen, die alternatives gesellschaftliches Denken und Handeln erzeugen, nicht zum eigenständigen Thema ihrer Untersuchungen machten, liegt offensichtlich an eben diesen Erfahrungen. Schon in der 48er Revolution stieß der revolutionäre Intellektuelle Marx, damals Chefredakteur der ,Neuen Rheinischen Zeitung", mit einer autonomen Arbeiterbewegung, dem Kölner Arbeiterverein, zusammen, weil dieser die Marxsche Wahlkampfstrategie, zusammen mit der liberalen rheinischen Bourgeoisie gegen den preußischen Absolutismus zu kämpfen, verwarf. Der Kölner Arbeiterverein war der Überzẹgung, daß die „revoluționäre Proletariatsparthei“ von keiner anderen Klasse als der eigenen etwas zu erwarten hatte, und schrieb die Aufgabe auf seine Fahne, „die Revolution permanent zu machen "(15). Vergleicht man die Marxsche Position zu dieser Frage, die er zwei Jahre später formulierte, so wird man feststellen, daß der Intellektuelle Marx von den nichtintellektuellen Arbeitern gelernt hatte. In der „Ansprache der Zentralbehörde an den Bund vom März 1850" schreiben Marx und Engels über die

9 Marx/Engels, Die heilige Familie, MEW 2, S. 89

10 ebenda, S. 55

11 Marx/Engels: Manifest der Kommunistischen Partei, MEW 4, S. 490

12 Marx an Ruge vom September 1843, MEW 1, S. 346

13 Marx: Das Elend der Philosophie, MEW 4, S. 143

14 Marx: Konspekt zu Bakunins Buch „Staatlichkeit und Anarchie“, MEW 18, S. 636

15 Freiheit, Arbeit. Wochenblatt. Organ des Arbeitervereins. Köln 1849. Reprint mit ergänzenden Anmerkungen von E. Czobel und Hans Stein. Glashütten im Taunus 1972, S. 52 
Aufgaben der deutschen Arbeiter: „,... sie selbst müssen das meiste zu ihrem endlichen Siege dadurch tun, daß sie sich über ihre Klasseninteressen aufklären, ihre selbständige Parteistellung sobald wie möglich einnehmen, sich durch die heuchlerischen Phrasen der demokratischen Kleinbürger keinen Augenblick an der unabhängigen Organisation der Partei irremachen lassen. Ihr Schlachtruf muß sein: Die Revolution in Permanenz"(16).

Auch die Reorganisation der deutschen Arbeiterbewegung nach der 48er Niederlage war das Werk der Arbeiter selber. Die „Leipziger Kongreßbéwegung" so nennt S. Na'ạman die Sammlung und Selbstverständigung der Arbeiter im sächsischen Raum zu Beginn der 60er Jahre - hatte sich herausgebildet und organisiert, bevor sie Intellektuelle aufforderte, ihre Ansichten über die Arbeiterbewegung, ihre Ziele und die Mittel, sie zu erreichen, zu äußern. Und sie orientierte sich nicht an Schulze-Delitzsch, obwohl dieser damals a1s ,König im sozialen Reiche“ galt, sondern an Ferdinand Lassalle. Und es gab eine stillschweigend vorausgesetzte Sprachregelung zwischen Lassalle und den Arbeitern. Lassalle mußte nicht die Grenzen der damals erlaubten politischen Diskussion übertreten, um sich verständlich zu machen. Er konnte darauf vertrauen, daß, sobald er "allgemeines Wahlrecht" sagte, die Arbeiter es als ,Revolution "verstanden, so wie er es explizit verstanden wissen wollte. Ein Zeuge verbürgt sich dafür: ,Alle Arbeiter, die ihn gehört, haben es auch so verstanden" (17). Für Karl Korsch fiel durch diese wohlverbürgte Sprachregelung „ein ganz ungeheures neues Licht" auf alles, ,was Lassalle über das ,allgemeine Wahlrecht" und verwandte Dinge jemals gesprochen und geschrieben hat "(18). Ich meine, daß dies gleichermaßen das Selbstverständnis der damaligen Arbeiter beleuchtet.

Auch die I. Internationale wurde nicht, wie die parteimarxistische Historiographie glauben machen will, von Marx gegründet, sondern von französischen und englischen Arbeitern. Und diese wollten durch eine internationale Arbeiterverbindung nicht nur unmittelbare Interessen durchsetzen, die soziale Lage durch Verkürzung der Arbeitszeit und Erhöhung der Löhne verbessern und dem internationalen Streikbrechertum einen Riegel vorschieben. Die englische Arbeiterschaft verhinderte sogar gegen ihre eigenen Interessen den Eingriff ihrer Regierung in den amerikanischen Bürgerkrieg zugunsten der Südstaaten. Dem polnischen Aufstand zu Hilfe zu kommen, war damals ein Ziel von Arbeiteraktionen. Und in einer Adresse der eng. lischen an die französischen Arbeiter stand die Forderung einer Vereinigung der 'Arbeiter aller Länder gegen die Ränke der Geheimdiplomatie (19).

Im 19. Jahrhundert gibt es viele Beispiele gegen die These, daß geschulte Intelligenz Voraussetzung für alternatives ge sellschaftliches Denken und Handeln sein soll. Vielmehr bestätigen sie die Auffassung, daß Arbeiter das, was sie sich

16 Marx/Engels: Ansprache der Zentralbehörde an den Bund vom März 1850, MEW 7, S. 254

17 zitiert bei Eduard Bernstein: Vorbemerkung, in: Ferdinand Lassalle: Gesammelte Reden und Schriften Bd. III. Berlin 1919, S. 176

18 Karl Korsch: Einleitung, in: Karl Marx: Randglossen zum Programm der Deutschen Arbeiterpartei. Leipzig 1922, S. 11

19 vgl. Julius Braunthal: Geschichte der Internationale Bd. 1, Hannover 1961, S, 101 ff. 
selbst zur Aufgabe machen, auch verstehen lernen und bewältigen können. Die Frage des revolutionären Subjekts, die Genese von Klassenbewußtsein war im 19. Jahrhundert bis zur Herausbildung der großen Arbeiterorganisationen überhaupt nicht Problem. Marx reflektierte das Verhältnis von revolutionärer Theorie und revolutionärer Praxis auch nicht, wie Bahro meint (226), auf dem Hintergrund einer Avantgarde, die aufgrund der Kenntnis der Theorie Ziele, Organisationsformen und Kampfstrategien wissenschaftlich abzuleiten und mit diesem Wissen die Massen zu erziehen, zu organisieren und ihren Kampf anzuleiten hätte. ,Kopf und Herz der Revolution" stellte er sich nicht auf zwei verschiedene gesellschaftliche Gruppen verteilt vor, sondern als zwei Momente, die in jedem Revolutionär vereinigt sein soliten. Theorie war überhaupt nur notwendig, weil es in der Marxschen Konzeption der sozialistischen Revolution nicht darum ging, ein Problem zu lösen, das die gesellschaftlichen Verhältnisse in Form sich zuspitzender Widersprüche stellten, sondern darum, die Philosophie zu verwirklichen, d. h. die gesellschaftlichen Verhältnisse, in denen die Menschen Objekte sind, umzuwälzen und neue Verhältnisse herzustellen, die vom Willen und Bewußtsein der Menschen abhängen. Weil die Menschen ihre Verhältnisse mit Bewußtsein regeln sollen, war für Marx das Bewußtsein eine Sache, die der Kämpfende sich aneignen mußte, wenn er auch nicht wollte (20). Der Blitz des Gedankens, sein Einschlag in den naiven Volksboden war notwendig, weil der Kämpfer selbst ein klares Bewußtsein davon haben muß, warum er eigentlich kämpft. Der Prozeß der Aneignung von revolutionärer Theorie durch das Proletariat war deshalb bei Marx ein Lernprozeß, der vor der Revolution oder spätestens in ihrem Verlauf stattfinden mußte. Die Menschen sollten sich mit und durch Veränderung der Verhältnisse selbst verändern. Daß die Begründer des wissenschaftlichen Sozialismus von einer Erziehung der Arbeiter durch ,Gebildete" überhaupt nichts hielten, belegen zudem zwei Briefe von Engels an Eduard Bernstein aus dem Jahre 1882. Engels erklärt dort, was er von der revolutionären Selbsttätigkeit der Arbeiter hält und wie er die Rolle der Führer einschätzt: ${ }_{\text {OE }}$ Es ist mir gar nicht bange um unsre deutschen Jungen, wenn's zum Klappen kommt. Jede Probe bestehn sie famos. Und nicht sie geben sich philiströs, es sind nur die Herren Führer, die von Anfang an von den Massen geschoben worden sind, statt die Massen zu schieben." (21) Und auch folgende Sätze kritisieren vorweg die später aufgestellte These vom ,induzierten Klassenbewußtsein ${ }^{66}$. Engels wendet sich dort gegen , die Leute, die ihr bißchen Bildung für absolut nötig halten, damit der Arbeiter sich nicht selbst befreie, sondern durch sie erlöst werde; Befreiung der Arbeiterklasse ist ihnen nur möglich durch den jebildeten Spießbürger; wie sollen die armen, hülflosen, unjebildeten Arbeiter das selbst besorgen!" (22)

Die These vom induzierten Klassenbewußtsein tauchte erst auf, nachdem die Arbeiterbewegung feste Organisationen entwickelt hatte und die Trennung von Theorie und Praxis in den Arbeiterorganisationen selbst institutionalisiert war. Erst als sich Intellek tuelle aus den Reihen der ,einfachen“ Arbeiter herauskristallisiert

vgl. Marx an Ruge vom September 1843, MEW 1, S. 345

21 Engels an Eduard Bernstein vom 22. Februar 1882, MEW 35, S. 278

22 Engels an Eduard Bernstein vom 13. September 1882, MEW 35, S. $360 \mathrm{f}$. 
hatten oder von außen dazugestoßen waren, und aufgrund ihres Wissens (d.h. der Fähigkeit, die Marxsche Theorie zu verstehen) die Ziele, Organisationsformen und Kampfstrategien „wissenschaftlich" erarbeiteten, den Massen also damit ihre Aufga" ben abnahmen, setzte sich das (Vor-)Urteil fest, diese selbst seien nur in der Lage, ein tradeunionistisches, also nur immanent kritisches Bewußtsein auszubilden. Erst nach der Jahrhundertwende erklärte Kautsky: ,Das modeme sozialistische Bewußisein kann nur erstehen auf Grund tiefer wissenschaftlicher Einsicht. ... Der Träger der Wissenschaft ist aber nicht das Proletariat, sondern die bürgerliche Intelligenz; in einzelnen Mitgliedern dieser Schicht ist denn auch der moderne Sozialismus entstanden und durch sie erst geistig hervorragenden Proletariern mitgeteilt worden, die ihn dann in den Klassenkampf des Proletariats hineintragen, wo die Verhältnisse es gestatten. Das sozialistische Bewußtsein ist also etwas in den Klassenkampf des Proletariats Hineingetragenes, nicht etwas aus ihm urwüchsig Entstandenes. Dementsprechend ... (ist es) ganz richtig, daß es zu den Aufgaben der Sozialdemokratie gehöre, das Proletariat mit dem Bewußrsein seiner Lage und seiner Aufgaben zu erfüllen" (23). Lenin übernahm diese These von Kautsky und zwar nicht nur als Beschreibung von Tatsachen, sondern als notwendige Erscheinung: ,wir haben gesagt, daß die Arbeiter ein sozialdemokratisches Bewußtsein gar nicht haben konnten. Dieses konnte ih. nen nur von außen gebracht werden." (24)

Aber selbst wenn Kautsky und Lenin die bewußtseinsmäßigen Kräfteverhältnisse zwischen den Arbeitern und der Intelligenz um die Jahrhundertwende richtig einschätzten, ignorierten sie die Tatsache, daß es auch unter dieser Voraussetzung immer noch zwei Alternativen gab, wie man die von Marx und Engels entwickelte sozialistische Theorie für die Emanzipation der Arbeiter umsetzen konnte. Es wäre auch angesichts eines nur tradeunionistischen Bewußtseins in der Arbeiterklasse möglich gewesen, das von Marx 1843 Ruge gegenüber entwickelte Programm durchzuführen, nämlich ,durch Analysierung des mystischen, sich selbst unklaren Bewußtseins" dieses zu reformieren, eine "Selbstverständigung der Zeit über ihre Kämpfe und Wünsche" herbeizuführen (25), den Arbeitern klar zu machen, daß die Losung nicht heißen konnte: „ein gerechter Lohn für ein gerechtes Tagewerk", sondern heiBen mußte: ,nieder mit dem Lohnsystem". Auf einer solchen Grundlage hätte es jedenfalls eine Chance gegeben, daß die Organisation der Kräfte und die Ausarbeitung der Kampfstrategien fuir die Befreiung das Werk der Arbeiter selbst sein konnte. Es war Marx, der in den Instruktionen für die Delegierten des Zentralrats der Interna. tionalen Arbeiterassoziation schrieb: „Es ist die Aufgabe der Internationalen Arbeiterassoziation, die spontanen Bewegungen der Arbeiterklasse zu vereinigen und zu verallgemeinern, doch nicht, ihnen irgendein doktrinäres System zu diktieren oder aufzudrängen “s (26). Und daß Marx sich hier nicht gegen irgendein „doktrinäres System" wendet, sondern primär gegen das „Diktieren und Aufdrängen", zeigt sein

23 Karl Kautsky: Die Revision des Programms der Sozialdemokratie in Österreich, in: Die Neue Zeit, Jg. 20, Bd.1. Stuttgart 1902, S. 79 f.

24 W. I. Lenin: Was tun? in: Werke Bd. 5, Berlin/DDR 1955, S. 385

25 Marx an Ruge vom September 1843, MEW 1, S, 346

26 Marx: Instruktionen für die Delegierten zu den einzelnen Fragen, MEW 16, S. 195 
Vorschlag zur „Untersuchung der Lage der arbeitenden Klasse aller Länder, unternommen von der Arbeiterklasse selbst“. Es geht dabei nämlich darum, daß die Arbeiter selber „durch die Initiative eines so großen Werks ... ihre Fähigkeit, ihr Geschick in die eigenen Hände zu nehmen", beweisen (27).

Marx" Nachfolger verstanden ihre Aufgabe anders. Statt den Arbeitern Begriffe und Theorien zu liefern, mit denen diese ihre eigenen gesellschaftlichen Erfahrungen und Wiinsche sozialistisch aufarbeiten und in selbstbestimmte Praxis umsetzen konnten, haben die marxistischen Theoretiker der Arbeiterbewegung die Theorie immer dazu benutzt, aus ihr ,notwendige "Organisationsformen und Strategien wissenschaftlich abzuleiten und diese als objektiv bestimmte Aufgaben den Arbeitern aufzuoktroyieren. Sie sorgten nicht praktisch für die Aufhebung der Trennung von revolutionärer Theorie und Arbeiterbewußtsein; sondern schrieben diese Trennung organisatorisch fest. Sie beanspruchten ein Monopol auf wissenschaftliche Interpretation der gesellschaftlichen Verhältnisse und der Ẻedingungen ihrer Veränderung. Ich bin mir bewußt, daß ich hier nur auf Plausibilitätsebene argumentiere, aber ich will die provozierende These wagen: Die Theoretiker der Arbeiterbewegung von Kautsky an haben die Massen, was das sozialistische Bewußtsein angeht, enteignet und zwar in doppelter Hinsicht. Sie haben ihnen die Aufgabe, alternative gesellschaftliche Bedürfnisse, alternatives gesellschaftliches Denken auszubilden, sozialistische Ziele, Organisationsformen und Kampfstrategien zu entwickeln, abgenommen. Sie haben das guten Gewissens getan, weil sie der Überzeugung waren, das alles könne man nur wissenschaftlich betreiben. Sie haben aber dann ihr Wissen nicht so angewandt, daß sie Theorien, Begriffe, Erklärungsmuster bereitstellten, mit deren Hilfe die Massen ihre gesellschaftskritischen Wünsche, Träume, Bestrebungen ,wissenschaftlich" aufarbeiten konnten, sondern sie haben aus der Theorie stellvertretend für die Massen Organisationsformen und Strategien wissenschaftlich abgeleitet, in denen die gesellschaftskritische Subalternität der Massen theoretisch und praktisch festgeschrieben wurde. Die Partei hat immer noch dieselben Aufgaben, seit Kautsky. Daß der ,marxistisch-leninistischen Theorie ${ }^{66}$ eine ,"wachsende Bedeutung ${ }^{66}$ in der ,Erziehung der Werktätigen“" zukommt, und daß deshalb ,nur die Partei, die die fortgeschrittenen Vertreter des Volkes vereint, die die gesellschaftlichen Entwicklungsgesetze kennt und über gewaltige praktische Erfahrungen bei der Gestaltung der neuen Gesellschaftsordnung verfügt, ... den Aufbau des Kommunismus wirklich wissenschaftlich leiten", ,die Aktivität der Massen ... organisieren und auf wissenschaftlich fundierte Ziele ... lenken " kann, ist noch in neuesten Publikationen über „wissenschaftlichen Kommunismus" nachzulesen (28). Und seltsamerweise kann sie diese Aufgabe offensichtlich nie erfüllen. Die Partei hat bereits Generationen erzogen, organisiert und geleitet. Und statt irgendwann einmal erledigt zu sein, wachsen diese Aufgaben. Und noch keiner der Erzieher ist auf die Idee gekommen, daß eine Emanzipation von den Erziehungs-, Organisations- und Leitungsberechtigten nottäte.

28 (Autorenkollektiv): Wissenschaftlicher Kommunismus. Berlin/DDR 1972, S. 426 
Man kann sicher Lenin nicht unterstellen, Sozialismus mit einer Erziehungsdiktatur der revolutionären Avantgarde verwechselt zu haben. Erziehung war seines Erachtens notwendig, um die ganze Klasse später unmittelbar an die Staats- und Wirtschaftsverwaltung heranlassen zu können (29). Die hier zur Debatte stehende Frage ist nur: Wann ist später? Man sollte meinen: sicher 1977 in der DDR. Aber Bahros strategisches Konzept einer Kulturrevolution impliziert dasselbe Verhältnis von Partei (Bund der Kommunisten) und den Massen. Seine Formulierungen sind verräterisch: Heißt es doch die politische Vertretung der emanzipatorischen Interessen betreffend: „Die Stelle ist vakant! ${ }^{66}$ und nicht: Sie ist überflüssig. Der alten Partei wird vorgeworfen, die ,Geschäfte verwechselt zu haben", und nicht, sie usurpiert zu haben (vgl. 424). Die neue Partei, obwohl nicht mehr von ,metaphy. sischer Notwendigkeit", ist aber immer noch ein ,unersetzliches Werkzeug" (413). Man fragt sich, in wessen Hand und zu wessen Bearbeitung. Auch das Problem ist noch das alte, nämlich das ,der Heranführung der Massen an die Kulturrevolution " (449). Der Bund der Kommunisten soll ,effektiv führen", soll ,gewährleisten ", soll sogar den Staatsfunktionen ,einen höheren Grad von Autorität verleihen, ihnen den freiwilligen Respekt der Öffentlichkeit sichen ${ }^{66}(430)$, so als hätte Bahro nie im ersten Kapitel seines Buches alle Argumente von Marx und Engels zur Kritik des Staates zusammengetragen und sich zu eigen gemacht.

Dabei kann man bei Bahro durchaus Einschätzungen finden, die meine Einwände gegen die Theorie und Praxis des induzierten Klassenbewußtseins bestätigen: Daß Fähigkeiten, die nicht genutzt werden, abhanden kommen, stellt Bahro bei seinem Rekurs auf die ökonomische Despotie der Inkas fest. Die Pflicht zu Gehorsam und Unterwerfung auf der einen Seite und die Verantwortung der Herrschenden, kein Individuum in materielle Not kommen zu lassen, auf der anderen Seite, führte zu einem Prozeß der ,historische(n) Disqualifizierung der unmittelbaren Produzenten, die in wenigen Generationen zu stumpfer Unmündigkeit und Initiativlosigkeit herabsanken, zu einem Zustand, wie er dem freien Mitglied einer archaischen Dorfgemeinschaft niemals eigen gewesen war" (90). Daß ein ,System der organisierten Verantwortungslosigkeit" auch heute noch die gleichen Auswirkungen auf die Fähigkeiten ihrer Mitglieder hat, merkt Bahro wenig später an: „lnzwischen haben spätestens die DDR und die CSSR bewiesen, daß̣ jeder beliebige Kommis in unserem System verlernen kann, so etwas zu machen", nämlich eigenständig eine Entscheidung zu treffen (vgl. 134).

Auch daß die Unfähigkeit und Untätigkeit der Massen in der Praxis der Avantgarde begriindet liegt, bestätigt Bahro indirekt. Gegen Rosa Luxemburgs Kritik an Lenin von 1918 wendet er nur ein, daß man erst einmal anerkennen müsse, ,daß die Rolle der Bürokratie als ,einziger Initiator' primär der Ersatz für die fehlende Masseninitiative war". Sekundär jedoch sei es keineswegs falsch, ,die Abwesenheit von Masseninitiative auf die Tätigkeit der Bürokratie zurückzuführen " (241). Und auch für meine Grundthese, daß die Menschen die notwendigen Fähigkeiten durchaus entwickeln können, wenn sie sich nur die Aufgabe selbst stellen und nicht zuschreiben lassen, gibt es einen indirekten Beleg bei Bahro. In der CSSR und in Polen

29 W. I. Lenin: II. Gesamtrussischer Verbandstag der Bergarbeiter, in: Werke Bd. 32, S. 51 
habe sich gezeigt, daß die Arbeiter, gerade dann eine progressive Rolle in der Gesellschaft spielen können, wenn sie sich von der Vormundschaft des Parteiapparats emanzipieren " (225).

Obwohl Bahro die Bewußtseinsverhältnisse im real existierenden Sozialismus als abhängige Variable der sozialen Organisation, der Ausbildung-und der gesellschaftlichen Arbeitsteilung interpretiert, mit der auch in einem kulturrevolutionären Prozeß wie mit einer Konstanten zu rechnen sei, weiß er auf der anderen Seite, daß die Trennung von Intelligenz und Arbeiterklasse in der bisherigen Arbeiterbewegung organisatorisch festgeschrieben wurde, also auch politisch, nicht nur sozialökonomisch produziert wurde: Wenn die Arbeiterklasse Intellektuelle hervorbrachte, seien diese vom Apparat absorbiert worden. Übrig blieben dann als ,Arbeiter" die Nichtintellektuellen, die mit einem subalternen, bloß tradeunionistischen Bewußtsein. Und das definierte sie dann. Genau diesen Prozeß und deren Folgen beschreibt Bahro, wenn er Folgendes ausführt: „Nicht die Arbeiterklasse gab sie (die Intellektuellen) sich als Führung, sondem sie (die Inteilektuellen) gaben sich der Arbeiterklasse als Führung. Und um einen Platz unter ihnen einnehmen zu können, mußten die Arbeiter selbst Intellektuelle werden, wobei die Tatsache der Arbeitsteilung und Klassenstruktur in der bürgerlichen Gesellschaft, die sich auch in den Arbeiterorganisationen widerspiegelt, stets zur Folge hat, daß diese Arbeiterintellektuellen aufhören, Arbeiter zu sein, als Arbeiter zu leben, daß sie in ein anderes Milieu, in eine besondere Existenz als Ideologen und Organisatioren, als, Offiziere der Bewegung überwechseln. Nach dem Sieg treten sie ihren Klassengenossen als Funktionäre des herrschenden Apparats gegeniber" (229). „Die intellektuelle Avantgarde nimmt eine solche Rolle bereits in der Fühung der politischen Arbeiterbewegung vorweg" (231).

Aber Bahro liefert nicht nur die Argumente zur Kritik seiner eigenen leninistischen Denkstrukturen, bei ihm finden sich auch die Ansatzpunkte für die Ableitung einer ganz anderen, emanzipativen Konzeption der Kulturrevolution. Im Kapitel 11 über das Potential für eine erneute Umgestaltung der Gesellschaft geht er typischerweise weder von der Klassengliederung in den Ländern des real existierenden Sozia. lismus, noch von deren sozialer Schichtung aus. Grundlage seiner Uberlegungen ist auch nicht mehr die Schichtung des Bewußtseinsniveaus, die Stufenleiter der Möglichkeiten, synthetisches Denken zu erlernen und gesellschaftlich anzuwenden. Hier geht es vielmehr um ,das Kräfteverhältnis zwischen dem überschüssigen und dem absorbierten Bewuftsein" (373), das zwar noch mit der sozialen Schichtung korreliert, aber offenbar anderen Bedingungen unterliegt. An dieser Stelle läßt Bahro sich auch nicht von den statistisch feststellbaren Fakten des status quo beeindrucken. Er stellt fest, daß dieses Kräfteverhältinis, insbesondere in der Form ,zwischen emanzípatorischen und Apparatinteressen, ... politisch viel ungleichgewichtiger in Erschę. nung (tritt), als es sozialökonomisch ist" (376). Die Tatsache, daß es keine Artikulationsmöglichkeit für die emanzipatorischen Interessen gibt und das absorbierte Bewußtsein durchgängig institutionalisiert ist im bestehenden System, macht die erscheinende Verteilung der Interessen zum Oberflächenphänomen (vgl. 376 f.). Am Grunde der Moldau, da rollen die Steine. „Das Subjekt der Emanzipationsbewegung 
findet sich in den energischen, schöpferischen Elementen aller sozialen Schichten und Bereiche“ (387). ,Das überschüssige Bewußtsein ist eben ein - wenn auch ungleich starkes - Kontinuum. Längst bringen auch die unmittelbaren Produzenten, zumindest durch ihren passiven Widerstand gegen das gesamte intellektuelle Personal der Leitung, Verwaltung, und oft auch Produktionsvorbereitung, für das sie meist summarisch den Terminus ,Bürokraten "verwenden, die tiefe Unzufriedenheit mit ihrer Rolle als subalterne Teilarbeiter zum Ausdruck, die ihrem Bildungsstand immer weniger entspricht" (386 f.). Und auch das Personal des Apparats düre man keineswegs mit diesem gleichsetzen (vgl. 397). In der CSSR 1968 sei klar geworden, „daß die-Mehrheit der aktiven Parteimitglieder auf einen Aufbruch zu neuen Ufern wartet" (362). Es sei nur eine Minderheit von Linienfunktionären in Partei, Staat und Wirtschaft, die sich in ihrem gesamten Lebensprozeß ließe. ,Diese und nur diese Leıte, die also auch subjektiv durchbürokratisiert sind, so daß ihnen nachher keine produktive Existenzform mehr offenstünde, sind jetzt die Feinde jeglicher Veränderung, weil sie unvermeidlich ihre Leidtragenden wer $§$ den. Sie allein sind die politbürokratische Reaktion. Und sie sind ideologisch so isoliert, daß sie zu ihrer Sicherheit ständig der latenten militärischen Intervention zu ihren Gunsten bedürfen" (387).

Das einzige Moment in diesem Kapitel, an dem Bahro die alte Parteikonzeption aufhängen zu müssen meint, besteht in der Beantwortung der Frage, bei wem zuerst das Bewußtsein von der Notwendigkeit der Veränderung zum Durchbruch kommt (vgl. 388). Aber wenn es auch richtig ist, daß die Bewegung in der CSSR nicht an der Basis ansetzte, sondem bei dem Personal des Ǔberbaus, und es ausreichende Gründe gäbe, diese Erfahrung zu verallgemeinern, daß nämlich die Wendung bei den Ideologen beginnt, ,und die Mobilisierung für die Reform ... als Kettenreaktion durch die Bildungsstruktur von oben nach unten ab(läuft)" (371), so spricht gerade die Tatsache der Kettenreaktion dafür, daß ein Erziehungsprozeß zur Herausbildung und Mobilisierung eines adäquaten kulturrevolutionären Bewußtseins völlig überflüssig ist. Eine Kettenreaktion ist ein Prozeß, für den das Potential bereits vorhanden ist, in dem die Bewegung nur noch des auslösenden Moments bedarf. Keineswegs muß dieses Potential erst noch herausgebildet werden. Der neue Bund der Kommunisten würde mit seinen vermeintlichen Erziehungsaufgaben offene Türen einlaufen, wenn nicht auf entrüstete Abwehr bei den Adressaten stoßen.

Das bedeutet natürlich nicht, daß .ein gesellschaftlicher Lernprozeß unnötig wäre. Aber diesen Lemprozeß müssen alle durchmachen, auch die progressiven Intellektuellen, denn „die lnteressen der ... managerialen, wissenschaftlichen und ideologischen Intelligenz tragen ebensowenig universalen Charakter wie die der unmittelbaren Produzenten " (239). Damit sagt Bahro selber, daß die Intellektuellen den unmittelbaren Produzenten nichts voraushaben, worauf sie eine Erziehungs- und Führungsposition in der Kulturrevolution gründen könnten. Sie alle müssen sich in einer praktischen Bewegung selbst verändern. „Die Kulturrevolution widerspräche sich selbst, wenn sie darauf hinausliefe, daß sich eine neue Avantgarde in dem alten administrativen Monopol auf Erziehung und Meinungsbildung festsetzte" (356).

Ich kann Bahros Festhalten an der These des induzierten Klassenbewußtseins 
und den daraus folgenden Aufgaben des Bundes der Kommunisten nur als Schwerkraft eines Paradigmas in seinem Denken begreifen. Es finden sich in seinem Buch nicht nur alle theoretischen Momente der Kritik dieser Revolutionskonzeption, sondern auch alle empirischen Bedingungen und die Umrisse einer alternativen Kulturrevolution.

\section{Bahros Regründungszusammenlang für eine erneute Umwälzung der gesell- schaftlichen Verhältnisse}

Auch was die Begründung der Notwendigkeit einer kulturrevolutionären Bewegung betrifft, gehen tradierte leninistische Interpretationsmuster in Bahros Argumentation ein.

Das beginnt mit seiner bewußten Anlehnung an die Marxsche ,Kapital" Analyse. Mein Einwand betrifft dabei nicht Bahros Anliegen, eine wissenschaftliche Analyse mit der Absicht praktischer Weltveränderung vorzulegen. Ich will auch nicht bestreiten, daß die sozialistischen Systeme in den osteuropäischen Ländern eigene Gesellschaftsformationen darstellen. Meine Bedenken betreffen vielmehr die Bahros Darstellung unausgesprochen zugrunde liegende. Annahme, der real existierende Sozialismus sei wie der Kapitalismus ein System, das sich als mit objektiver Eigengesetzlichkeit begabter $Z$ wangszusammenhang hinter dem Rücken der Menschen herstelle, reproduziere und dabei eine immanente Entwicklungsdynamik entfalte, ein System, das ,seine eigenen Gesetze" habe (55), das demnach aus seiner „eigenen Gesetzmäßigkeit erklärt werden müsse" (14 f.) und von dem dann auch ein ,allgemeiner Begriff" entfaltet werden könne (30).

Noch problematischer aber erscheint mir Bahros Versuch, die Notwendigkeit der Kulturrevolution objektiv aus der Analyse der bestehenden sozialistischen Systeme abzuleiten. „Die Veränderungen werden ausgehen von den objektiven Widersprüchen " (389), stellt er fest. Aber was heißt es konkret, wenn er behauptet, die Krise des real existierenden Sozialismus ,beruht letztlich auf der Zuspitzung des ... Widerspruchs zwischen den modernen Produktivkräften und den zum Hemmnis für sie gewordenen Produktionsverhältnissen" ( $11 \mathrm{f}$.). Wie ist seine These zu begreifen, daß ,die vergegenständlichten Produktivkräfte unwiderstehlich nach Wiederherstellung der gesamtgesellschaftlichen Kooperation drängen, während die alte Arbeitsteilung noch triumphiert " (174)?

Zunächst kann man zeigen, daß Bahro seiner eigenen Behauptung, daß die Verhältnisse des real existierenden Sozialismus sich unabhängig vom bewußten und gewollten Handeln der Menschen durchsetzen, von ,niemandes Willkür erzeugt " seien (43), selber widerspricht; denn er stellt fest, die obere Oligarchie unterwerfe ,den ganzen Reproduktionsprozeß des ökonomischen, sozialen, kulturellen Lebens ihrem Reglement" (284). Das heißt doch nichts anderes, als daß die gesamte gesellschaftliche Struktur durch die Köpfe der Herrschenden vermittelt ist, daß sie sich

30 vgl. Willfried Spohn: Geschichte und Emanzipation, Bahros Beitrag zur Sozialismü-Diskussion, in: Prokla Nr. 31, Berlin 1978, S. 21 
also nicht hinter deren Rücken selbst vermittelt. Auch ist die Partei der Motor und der Steuermann der sozialen Entwicklung (vgl. 286). Sie steht zwar in dieser Funktion in einem Zwangszusammenhang. Dessen Druck ist aber kein Binnendruck der eigenen Gesellschaft. Das Bewegungsgesetz des real existierenden Sozialismus wird vielmehr diktiert durch die kapitalistische Entwicklung, ihre Herausforderung und ihre Bedrohung. Bahro selbst stellt fest, daß die Maßstäbe der Akkumulation in den Ländern des real existierenden Sozialismus durch die Konkurrenz mit dem Kapitalismus gesetzt werden (vgl. 156), daß das ,Prinzip der Profitmaximierung ... mächtig in den real existierenden Sozialismus hinein regiert" (310) nach dem Motto aus dem Märchen: „Der lgel ist schon da“".

Wenn Bahro aber das gesellschaftliche Handeln in den sozialistischen Systemen nicht als einen objektiven $Z$ wangszusammenhang ausweisen kann, kann er auch nicht objektiv erklären, welche gesellschaftlichen Zusammenhänge notwendig zu der Verlarvung des Systems geführt haben, d. h. er kann die Dimensionen nachkapitalistischer Praxis nicht benennen, die den, ,Uberapparat" hervorbrachten. Die Fortdauer der gesellschaftlichen Arbeitsteilung ist dafür ja nicht die Ursache. Diese muß, so Bahro, auch nach der Kulturrevolution fortbestehen. Auch die anhaltende Subsumtion der Individuen unter die Arbeitsteilung, die durch die fortgesetzte Trennung von Hand- und Kopfarbeit weiterbestehende Subalternität kann nicht causa sein. Diese Momente liegen zwar der Existenz des Apparats zugrunde, aber sie reproduzieren ihn nicht zwangsläufig. Sondern umgekehrt: Der Apparat verhindert den möglichen gesellschaftlichen Lernprozeß und damit die Aufhebung der Subalternität.

Bahros Analyse ist eigentlich nur deskriptiv. Seine Kritik des Bestehenden beschränkt sich darauf, das System als „Apparat", die konservativen Interessen als „Apparatinteressen“" zu kennzeichnen, eine Verdoppelung des Staatsapparats in eine Partei- und eine Verwaltungsbürokratie zu konstatieren. Er stellt Mutmaß3ungen an über ein ,Wesen der Institutionen ", welches offenbar zwangsläufig in die Verbürokratisierung hineinführt (vgl. 134), aber aufgrund von Momenten der „Trägheit“ (16) , "Erstarrung“ (138), Vergreisung (vgl 160) etc. nicht wieder hinausführt (vgl. seine Argumentation S. 383 ff.). Oder er rettet sich in das Gebiet der Psychologie und stellt Karrierismus (252), „Kasteninteressen" (13), Machtstreben und Selbstbehauptungswillen der höchsten Funktionäre (vgl. 297) und deren sozialpsychologische Regression (vgl. 160) fest. Aber selbst eine solche Art der Erklärung steht auf schwankendem Boden, da Bahro an anderer Stelle zeigt, daß es letztlich nur eine Minderheit von subjektiv völlig durchbürokratisierten Linienfunktionären ist, welche die Feinde jeglicher Veränderung ausmachen (vgl. $377 \mathrm{f}$.). Und die Gesellschaft gehorche allein der Gewalt, die jene Minderheit loszulassen vermöge (vgl. 378).

Wenn Bahro nicht in der Lage ist, das System des real existierenden Sozialismus als einen objektiven gesellschaftlichen Zwangszusammenhang darzustellen, dem eine eigengesetzliche Dynamik inhäriert, dann kann er auch auf keine systemimmanent produzierte objektive Dialektik der revolutionären Veränderung verweisen. Bahro hat für eine lange Aufbauperiode bestätigt, daß aus objektiven Grïnden nicht der Sozialismus sondern die Industrialisierung Aufgabe war; und er hat die 
Notwendigkeit der bürokratischen Diktatur in dieser Phase anerkannt. An den objektiven Grundlagen für diese Einschätzung hat sich jedoch auch im weiteren Verlauf nichts geändert: Der kapitalistische Industrialisierungsdruck ist nicht gewichen, und auch die Notwendigkeit einer Institution zur Herstellung der gesellschaftlichen Synthese bleibt - Bahro selbst zufolge - weiter bestehen. Deshalb dienen ja gerade diese Gründe den status-quo-Apologeten als Argumente. Bahro kann also keine objektiven dem System und seiner Dynamik selbst entspringenden Gründe für die Notwendigkeit der Kulturrevolution angeben.

Und noch eine Konsequenz ergibt sich aus Bahros Anlehnung an marxistischleninistische Theoriebildung: Wenn er nicht objektiv erklären kann, welche gesellschaftlichen Zusammenhänge notwendig zur Verlarvung des Systems geführt haben, kann er auch seine These nicht begründen, weshalb die herrschende Klasse die bürokratischen Strukturen zu durchbrechen und eine Kulturrevolution zu initiieren micht in der Lage ist. Dann kann er aber auch keine Gründe angeben, weshaib ein Bund der Kommunisten diese Aufgabe bewältigen könnte, ohne wieder, wie die alten kommunistischen Parteien, in jenem, ,Teufelskreis der alten Arbeitsteilung "sich festzufahren (179). Geht dieser kommunistische Bund doch nach Bahro mit seiner kulturrevolutionären Bewegung von qualitativ ähnlichen Voraussetzungen aus: gesellschaftliche. Arbeitsteilung, die Notwendigkeit einer Institution für die Synthese der gesellschaftlichen Gesamtarbeit, subalterne Massen und die Bedrohung durch die kapitalistischen Systeme. Wenn ,aufgeklärte Minderheiten stets repressive Bürokratien geschaffen haben" (91), wenn ,sich das ,Wissen", wo es herrscht, stets ais bürokratische Hierarchie mit der Tendenz zur despotischen Spitze organisiert" hat (91 f.) - welche Erfolgsgarantie bietet dann ein Emanzipationskonzept, welches dieselben Strukturen in der Ausgangssituation aufweist? Bahro weist die Deformationstheorien zuriick, die auf der Überlegung aufbauen: „Wenn die Menschen, speziell die bolschewistischen Parteimenschen, nur intensiver gewollt und weiser gehandelt hätten, wäre statt des real existierenden Sozialismus der Sozialismus da, wäre er zumindest auf anderem, besserem Wege" (163). Aber Bahro selber hat auf dem Hintergrund der oben dargestellten bewußt objektivistischen Argumentation keine bessere Theorie anzubieten als die Hoffnung auf bessere Sozialisten.

Dabei entgeht ihm durchaus nicht, daß die Intelligenz, die den Bund der Kommunisten ausmachen soll, ebensowenig universalen Charakter trägt wie die unmittelbaren Produzenten (vgl. 239). Im Gegenteil: „Die bestehende Arbeitsteilung bewirkt, daß viele Wissenschaftler dazu neigen, ihre menschlichen Interessen allzu weitgehend in ihren scientifischen Interessen bzw. in ihrer Konkurrenz um die Anerkennung in der Fachwelt aufgehen zu lassen. Das ist ein ebensolcher Partikularismus wie der der anderen Interessengruppen auch, aber gerade in den besonderen Ambitionen der durch die Arbeitsteilung privilegierten Gruppen haben wir es zwangsläufig mit dem exponiertestén Rest der alten Klassenherrschaft zu tun " (419). Bahro selber wirft die Frage auf, ,ob bei einem solchen Aktivierungsmuster etwas anderes herauskommen kann, als bloß eine neue Machtverteilung zugunsten der Intellektuellen, der Wissenschaftler und der Wirtschaftsleiter" (389).

Bahros traditionell marxistischer Versuch, die objektiven und subjektiven 
Voraussetzungen einer allgemein menschlichen Emanzipation aus der wissenschaftlichen Analyse der bestehenden Verhältnisse abzuleiten, bringt $\mathrm{m}$. E. ebenso wenig, wie alle gleichartigen Versuche in der Geschichte der sozialistischen Theorie bisher gebracht haben: Alle Beweise der objektiven Notwendigkeit einer sozialistischen Revolution waren bisher für eine wirkliche sozialistische Revolution irrelevant, solange die Systeme dem für unausweichlich prognostizierten Zusammenbruch auf irgendeine Weise entgehen konnten. Nicht auf sich zuspitzende Widersprüche, für die es immer auch systemimmanente Lösungen gibt, kann man bauen. Vielmehr ist an den Widerspruch der Massen gegen die Systeme anzuknüpfen, mit ihm hat Theorie sich zu identifizieren, wenn die $_{8}$ Emanzipation der Menschen aus Herrschaftsverhältnissen schlechthin auf ihrem Programm steht (31). Die Funktion, die der Theorie dann zukommt, ist Kritik der gesellschaftlichen Verhältnisse durch Darstellung, ist Aufklärung darïber, daß das Unbehagen, die Beschränkungen, die Grenzen der Selbstverwirklichung gesellschaftlichen Charakter tragen. Kein System entwikkelt Widersprüche, die den Menschen objektiv die Aufgabe der Emanzipation stellen. Emanzipationsbedürfnisse gehen von Menschen aus, die sich nicht abfinden können mit dem, was das System sie zwingt zu sein. Deshalb muß die theoretische Reflexion an diesen Wünschen und Zielen der Menschen anknüpfen. Genau das tut Bahro auch: Seine Ökonomik der Kulturrevolution ist nicht als Lösung objektiver gesellschaftlicher Probleme, als Lösung des, Widerspruchs zwischen den modernen Produktivkräften und den zum Hemmnis für sie gewordenen Produktionsverhältnissen " $(11$ f.) konzipiert. Diese Ökonomik der Kulturrevolution - Umverteilung der Arbeit, einheitlicher Bildungsweg für voll sozialisierte Menschen, Bildungsfähig. keit und Lernmotivation der Kinder, ein neues Gemeinschaftsleben und Vergesellschaftung (Demokratisierung) des allgemeinen Erkenntnis- und Entscheidungsprozesses (450) - ist eine mögliche Lösung des Widerspruchs zwischen menschlichen Lebensbedürfnissen und ihren aktuellen, aufgezwungenen Lebensformen. Sie ist eine gesellschaftliche Alternative, eine Utopie, und damit keine wissenschaftlich abgeleitete objektive Aufgabe, sondern ein Vorschlag zur Diskussion, zur Selbstverständigung der Individuen über das, was sie wollen.

Zielorientiert und nicht auf der Grundlage objektiver Ausgangstatsachen wissenschaftlich abgeleitet sind auch Bahros Vorschläge zur Organisation der Kommunisten. Bahro hat eine radikale ,Reformation" (412) der Partei im Auge, deren qualitativ neue Aufgaben eine qualitativ neue Struktur erfordern. Er gèht sogar so weit, das Gelingen der Kulturrevolution von eben dieser ,Reformation der Partei“" abhän. gig zu machen (vgl。 444). Dabei geht es nicht nur um eine in der Parteiverfassung festgelegte vollständige Informations- und Diskussionsfreiheit und um unbeschränkte Basisaktivität. Vielmehr ist die konzipierte Struktur des Bundes der Kommunisten so angelegt, daß schon im Ansatz die Möglichkeit einer erneuten Trennung von Intellektuellen und unmittelbaren Produzenten, von Trägern sozialistischen (synthetischen) Bewußtseins und tradeunionistischen (subalternen) Bewußtseins verhindert wird. Denn Bahro will die Intellektuellen nicht als traditionelle Schicht oder Grup- 
pe, auch nicht als Inhaber von geistig synthetischen Fähigkeiten organisieren, sondern als Menschen, die erkannt haben, daß die Schranken ihrer Selbstverwirklichung gesellschaftlichen Charakter tragen (vgl. 433). Er will emanzipatorische Interessen organisieren, die vertikal durch die ganze Gesellschaft, quer durch die Bildungspyramide hindurch gehen. „, Deshalb muß der Bund der Kommunisten allen offenstehen, die das Bedürfnis haben, über die Verfolgung ihrer unmittelbaren Interessen hinauszugehen" " $(433)$.

Aber nicht nur im organisatorischen Ansatz wird ein Dominanzverhältnis zwischen "Führern" und "Geführten" ausgeschlossen. Bahro destruiert auch das Mittel, mit dessen Hilfe sich das Herrschaftsverhältnis auch in Gemeinschaften von ,Gleichen " immer wieder eingeschlichen hat, nämlich das alte Vorurteil von der Notwendigkeit der Disziplin bei der Durchführung einer gemeinsamen Aufgabe. „Wenn die Aufgabe, die Subalternität zu liquidieren, ihre Reproduktionsquellen zu beseitigen, richtig gestellt ist", so Bahro, „dann muß man sich innerhalb der Partei unbedingt von der Glorifizierung der proletarischen Disziplin lösen, die Lenin von Kautsky übernahm, weil sie auf die russischen Verhältnisse paßte" (439). Diese Wertschätzung der Disziplin sei von den Stabsbedürfnissen einer auf feindlichem Terrain kämpfenden Truppe diktiert gewesen, die keine Zeit zu verlieren hatte. ,Unter den gegenwärtigen Verhältnissen im real existierenden Sozialismus haben die intellektuellen Elemente der verschiedenen sozialen Schichten und Gruppen allen Grund, sich nicht unter Zeitdruck setzen zu lassen" (439 f.). Es gebe keinen Grund, die Beziehungen innerhalb des Bundes der Kommunisten oder die zwischen Kommunisten und dem Volk ,in Begriffen von Subordinationsverhältnissen " (443) zu beschreiben.

Auch einige in der ,Ökonomik der Kulturrevolution" beschriebene Sofortmaßnahmen wenden sich gegen eine mögliche Gefahr einer erneuten Herausbildung von Klassen und Klassenprivilegien während und nach der Kulturrevolution: Sollen diese doch dazu dienen, ,die Kluft der Sprachlosigkeit zwischen dem politischen Überbau und den Massen, den Interessengegensatz zwischen der wissenschaftlichtechnisch-ökonomischen Intelligenz und den Produktionsarbeitem in den Betrieben erst einmal zu überbrücken, mindestens die Fronten fließend zu machen " (456). Da$z u$ ist nach Bahro die Egalisierung der materiellen Lebensbedingungen notwendig. Führer, Aktivisten und Funktionäre müssen die durchschnittlichen Lebensbedingungen des Volkes teilen, d. h. sie können weder, was die Gehälter und sonstige Versorgungseinrichtungen betrifft, Sonderansprüche stellen, noch können sie sich vollständig von einfacher ausführender Arbeit ausnehmen. Es soll sich materiell nicht mehr lohnen, zur Vorhut der kulturrevolutionären Bewegung zu gehören.

Im Osten nichts Neues? In Bahros Buch findet man die traditionell leninistische These vom restringierten gesellschaftlichen Bewußtsein der unmittelbaren-Produzenten. Bahro versucht auch in einem Strang seiner Argumentation, die subjektiven und objektiven Bedingungen einer gesellschaftlichen Umwälzung aus der Analyse der bestehenden Verhältnisse abzuleiten. Ebenso formuliert er die Konsequenz aus beiden Thesen: Nur eine intellektuelle Elite sei befähigt, eine neue Gesellschaftsordnung zu antizipieren und die Kräfte für den Kampf gegen das alte System zu organi- 
sieren. Aber beide Thesen und auch deren Konsequenz sind keine tragenden Bestandteile von Bahros Reflexion über eine kulturrevolutionäre Strategie in den Ländern des real existierenden Sozialismus. Untersucht man seine These vom restringierten Bewußtsein der unmittelbaren Produzenten, auf welche Bahro die Notwendigkeit erneuter Erziehungs-, Organisations- und Führungsaufgaben des Bundes der Kommunisten gründet, so zeigt sich, daß seine Argumente eher die entgegengesetzte These stütžen, daß nämlich die Massen für die kulturrevolutionäre Praxis einer solchen pädagogischen Vorbereitung durchaus entraten können. Überprüft man den von Bahro dargestellten Begründungszusammenhang für die Notwendigkeit der Kulturrevolution, so wird deutlich, daß Bahro in seiner Konzeption von Organisation und Strategie sich nicht von zu lösenden objektiven Systemwidersprüchen leiten läßt, sondern an Emanzipationsbedürfnisse der Menschen, also an zu erreichende Ziele anknüpft.

Bahros Analyse des real existierenden Sozialismus ist eine Aufforderung zur Selbstverständigung der Individuen über die gesellschaftlichen Wurzeln und den gesellschaftlichen Charakter der Grenzen ihrer Selbstverwirklichung und eine Grundlage zur Diskussion alternativer gesellschaftlicher Verhältnisse, die diese Grenzen der Selbstverwirklichung sprengen. Damit knüpft Bahro wie schon einige in der DDR in den 50er Jahren, wie die Budapester Schule in den 60er Jahren an den emanzipativen Gehalt der Marxschen Theorie an. Und diese emanzipative Dimension ist genau der Strang Marxscher Theorie, der im traditionellen Leninismus ausgeblendet wurde. 